\title{
Characterization of polymer materials based on structure analyses of vicinal water
}

\author{
Hiromi Kitano
}

In this paper, we review the vibrational spectroscopic analyses of the structure and hydrogen bonding of water in the vicinity of various polymers based on research performed by our group. Aqueous solutions of water-soluble polymers have been examined using the contours of $\mathbf{O}-\mathbf{H}$ stretching in polarized Raman spectra. For water sorbed into polymer thin films, infrared spectroscopy was used and the structure of water at polymer-liquid water interfaces was examined using sum frequency generation spectroscopy. Zwitterionic polymers and amphoteric polymers containing comparable numbers of anionic and cationic groups were inert to the structure of vicinal water. In contrast, lopsidedly charged polymers strongly perturbed the structure of water. The inert properties of polymers with comparative contents of anionic and cationic groups with respect to the hydrogen-bonded network structure of water may be owing to the counteraction of the electrostatic hydration effect by the proximity between the cationic and anionic groups, which may endow the charge-neutralized (zwitterionic or amphoteric) polymers with biocompatibility. Similar inert properties to vicinal water were observed for a thin film of a well-known anticoagulant polymer (i.e., poly(2-methoxyethyl acrylate)). The correlation between the freezing/melting behavior of water sorbed into various nonionic polymer materials and their biocompatibility is also discussed.

Polymer Journal (2016) 48, 15-24; doi:10.1038/pj.2015.70; published online 16 September 2015

\section{INTRODUCTION}

Water is a very common and abundant compound with a simple structure, and it cannot be completely removed from materials on Earth. The effect of water on polymer materials is very important from scientific and industrial points of view. For example, the conformation of naturally occurring polymers (e.g., proteins, polysaccharides and polynucleotides.) in an aqueous solution minimizes the surface free energy of the polymers. Therefore, as Anfinsen demonstrated, the primary structure of a protein determines the tertiary structure. ${ }^{1}$ In addition, the thermoresponsiveness of various polymers, such as poly ( $N$-isopropylacrylamide) and poly[ $N$-(3-ethoxypropyl)-acrylamide], is due to the dehydration of polymers above a critical temperature (i.e., lower critical solution temperature). ${ }^{2}$

However, for useful polymers, the effect of water is typically estimated from the influence of water on the mechanical, physical and chemical properties of polymer materials. We have been characterizing polymer materials from the opposite viewpoint (i.e., structure analyses of water in contact with polymer materials) because it is highly probable that the properties of the polymer materials affect the structure and properties of vicinal water. Therefore, it is possible to obtain valuable information for the development of polymer materials by structure analyses of vicinal water. Water can be used as a 'probe' to characterize polymer materials.

The heat of solidification, heat of vaporization and heat capacity of liquid water are the largest among ordinary liquids. These anomalous physical properties are due to hydrogen bonding between the hydrogen atom of the water molecule and oxygen atom of a neighboring water molecule. ${ }^{3}$ In addition, the nonlinear bent structure of a water molecule results in a fluctuating hydrogen bond network in the liquid state. Vibrational spectroscopic methods (abbreviated as VSs hereafter) including Raman and infrared spectroscopies have been extensively used to analyze the so-called V-structure (vibrationaveraged structure) of water to determine the orientation of the water molecules because observation times $\left(\tau=10^{-13} \sim 10^{-14} s\right)$ that are shorter than the relaxation times of the rotational rearrangement $\left(\tau_{\mathrm{R}}\right)$ of the water molecules in the liquid phase $\left(\tau=10^{-11} \sim 10^{-12} \mathrm{~s}\right)$ are used in these methods. ${ }^{4}$ In addition, the vibrational relaxation time of the chemical bonds used as a probe in VSs is shorter than the average lifetime of the hydrogen bond between water molecules (in the order of $10^{-12} \mathrm{~s}$ ). Therefore, both the changes in the hydrogen-bonded network structures between water molecules and the rotational rearrangement of water molecules can be detected by VSs. However, other techniques (i.e., differential scanning calorimetry (DSC), X-ray diffraction, dielectric dispersion and NMR), which utilize longer observation times $\left(\tau \cong 10^{1}, 10^{1}, 10^{0} \sim 10^{-11}\right.$ and $10^{-6} \sim 10^{-11} \mathrm{~s}$, respectively) than the $\tau_{\mathrm{R}}$ or lifetime of the hydrogen bonds between water molecules, can detect the state of water based on the diffusion property of the water molecule (D-structure, diffusion-averaged structure). Therefore, changes in the structure of water cannot be directly detected using these techniques.

On the basis of this knowledge, we have been using vibrational spectroscopy, which is sensitive to the position and orientation of 
water molecules, for the analyses of water in the vicinity of polymer materials, and we have tried to clarify the correlation between the structure of vicinal water and the chemical properties of polymer materials. In this review, the experimental results that have been primarily obtained by our group are discussed with reference to related works by other groups. Raman spectroscopy and infrared spectroscopy have been used to analyze the structure of water in aqueous polymer solutions and polymer thin films, respectively. The changes in polarizability due to molecular vibrations are detected by the former method, and the changes in the dipole moment due to molecular vibrations are detected by the latter method.

For quantitative analyses of aqueous solutions, infrared spectroscopy cannot be easily used because water intensely absorbs infrared light, and the path length must reproducibly be $\sim 5 \mu \mathrm{m}$ in the transmission absorption measurements. The structure of the soluteinduced hydration shells was examined by attenuated total internal reflectance (ATR) with a path length of $\sim 7.5 \mu \mathrm{m} .{ }^{5}$ In contrast, Raman spectroscopy can be easily applied to an aqueous solution. The sum frequency generation (SFG) technique, which is a highly interfacesensitive technique, has been used to observe the structure of water at the polymer-liquid water interface.

\section{STRUCTURE ANALYSES OF WATER IN AQUEOUS SOLUTIONS CONTAINING WATER-SOLUBLE POLYMERS}

The $\mathrm{O}-\mathrm{H}$ stretching Raman band of liquid water is very broad and consists of several overlapping components. Walrafen decomposed the band into four Gaussian components and interpreted the band as being because of an equilibrium between the five-molecule, fully hydrogen-bonded structure with intermolecular $\mathrm{C}_{2 \mathrm{v}}$ symmetry and nonhydrogen-bonded monomeric water. ${ }^{6}$ Georgiev et al. ${ }^{7}$ noted a limitation in the decomposition method due to the required preliminary assumption concerning the number and shapes of the components.

New insight into the analyses of the $\mathrm{O}-\mathrm{H}$ stretching band of water was reported by Green et al. ${ }^{8}$ They analyzed the structure and hydrogen bonding of water in various types of aqueous solutions using the $\mathrm{O}-\mathrm{H}$ stretching contours of the polarized Raman spectra (Figure 1). According to their procedures, the intensity of the collective band $\left(I_{\mathrm{c}}\right)$ observed at $\sim 3250 \mathrm{~cm}^{-1}$ in aqueous polymer

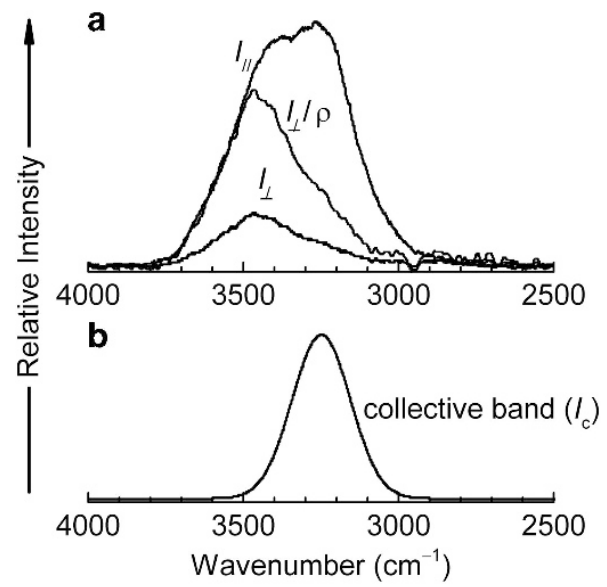

Figure 1 (a) $I_{/ /}$and $I_{\perp}$ spectra of pure water at $25^{\circ} \mathrm{C} . I_{\perp} / \rho$ spectrum is also shown ( $\rho$, degree of depolarization). (b) Collective band of water $\left(I_{/ /}-I_{\perp} / \rho\right)$. solutions was separated from the spectra using equation (1).

$$
I_{\mathrm{c}}=I_{/ /}-I_{\perp} / \rho_{\mathrm{O}-\mathrm{H}},
$$

where $I_{/ /}$and $I_{\perp}$ are the intensities of the spectra observed with the polarizer oriented parallel and perpendicular, respectively, to the incident laser beam and $\rho$ is the depolarization ratio, which indicates the symmetry of the vibration mode and is expressed by $\rho=I_{\perp} / I_{/ /}$. The $\rho$ value was set to extract the intensity of the collective band $\left(I_{\mathrm{c}}\right)$ by minimizing the contribution of the high frequency region of the spectra obtained in the parallel position.

Because the intensities of the Raman spectra are not absolute, the area of $I_{c}$ was normalized to determine the relative intensity of the collective band ( $C$ value) corresponding to the long-range coupling of $\mathrm{O}-\mathrm{H}$ stretching in the Raman spectra for aqueous solutions of various water-soluble polymers (equation (2)):

$$
C=\int I_{\mathrm{c}}(w) \mathrm{dw} / \int I_{/ /}(w) \mathrm{d} w,
$$

where $w$ is the Raman shift in $\mathrm{cm}^{-1}$.

In aqueous solutions, the water molecules interacting with hydrophobic or hydrophilic solutes change their positions and orientations in the hydration shell around the solutes. Therefore, the intensities of the collective band of aqueous solutions at a certain temperature $T$ $\left(C_{\mathrm{x}}(T)\right)$ increase or decrease due to the formation or dissociation of water-water H-bonding compared with that of pure water $\left(C_{\mathrm{w}}(T)\right)$ at the same temperature. The probability $\left(P_{\mathrm{d}}\right)$ that an $\mathrm{O}-\mathrm{H}$ oscillator is excluded from the H-bonded network of water molecules owing to an unfavorable orientation or position is defined in equation (3).

$$
P_{\mathrm{d}}=\frac{C_{\mathrm{w}}(T)-C_{\mathrm{x}}(T)}{C_{\mathrm{w}}(T)}
$$

The number of hydrogen bonds collapsed by the presence of one monomer residue of a polymer or one molecule of solute ( $N$ value) is estimated as

$$
N=P_{\mathrm{d}} / f_{x}
$$

where $f_{x}$ is the number of monomer units per one $\mathrm{O}-\mathrm{H}$ oscillator. ${ }^{9-12}$

It is important to note that an intrinsic defect in $\mathrm{H}$-bonding in pure water exists at a certain temperature compared with that of perfectly $\mathrm{H}$-bonded ice (for example, $C=0.365$ for pure water at $25^{\circ} \mathrm{C}\left(C_{\mathrm{w}}\right)$; $C=0.54$ for perfectly H-bonded ice $\left.\left(C_{\text {ice }}\right)\right) .{ }^{8}$ Although this factor should be considered for the evaluation of $P_{\mathrm{d}}$ values, the $P_{\mathrm{d}}$ values are calculated using the ratio of $\left(C_{\mathrm{w}}-C_{\mathrm{x}}\right)$ and $C_{\mathrm{w}}$ (equation (3)). Because the number of $\mathrm{O}-\mathrm{H}$ oscillators $\mathrm{H}$-bonded in pure liquid water at a certain temperature is smaller than the total number of $\mathrm{O}-\mathrm{H}$ oscillators, the $N$ values in equation (4) were simply corrected by this factor as $N_{\text {corr }}=N \times\left(C_{\mathrm{w}} / C_{\text {ice }}\right) .{ }^{13}$ However, the $N_{\text {corr }}$ value does not precisely indicate the actual number of $\mathrm{H}$-bonds that each group of polymers perturbs. This value represents the degree of perturbation of the H-bonded network structure of water because several assumptions were included during the derivation of the $N_{\text {corr }}$ value.

Table 1 shows the $N_{\text {corr }}$ values for various water-soluble polymers. The molecular weights of these polymers were small to exclude the formation of a 'pseudo-network' of polymer chains that perturbs the hydrogen-bonded network structure of surrounding water molecules in a manner similar to that of polymer gels with a high degree of cross-linking, ${ }^{9}$ and the following trends were observed.

(1) The $N_{\text {corr }}$ values for ionic polymers, such as sodium poly(acrylic acid) (NaPAA), sodium poly(ethylene sulfonic acid) (NaPES), poly (allylamine hydrochloride) (PAA.HCl) and poly(L-lysine hydrobromide) (PLL.HBr), were much larger than those of water-soluble 
neutral polymers, such as poly(ethylene glycol) and poly(1-vinyl-2pyrrolidone). ${ }^{10}$ These results indicate that the monomer residue of water-soluble neutral polymers does not substantially disturb the water structure. However, electrostatic hydration by the polyion has a substantial effect on the structure of the surrounding water molecules because they are quite polar.

(2) The $N_{\text {corr }}$ values for zwitterionic polymers, such as poly (2-methacryloyloxyethyl phosphorylcholine) (poly(phosphobetaine) PMPC), ${ }^{11,13}$ poly(3-sulfo- $N, N$-dimethyl-(3-methacrylaminopropyl) propanaminium inner salt) (poly(sulfopropyl betaine), PSPB $)^{12}$ and poly(2-carboxy- $N, N$-dimethyl-(2-methacryloyloxyethyl)methanaminium inner salt) (poly(carboxymethyl betaine), PCMB) (Scheme 1), ${ }^{14}$ and polymers possessing both cationic and anionic side groups (amphoteric polymers), such as poly[methacrylic acid-co-[(3-dimethylamino)propyl methacrylamide]] (poly(MA-co-DMAPMA)) (Figure 2) and poly[methacrylic acid-co-(3-methacryloylamino)propyl trimethylammonium chloride] (poly(MA-co-MAPTAC)) were not large, indicating that these polymers do not significantly disturb the hydrogen-bonded network structure of water. ${ }^{15,16}$ In the copolymer

Table $1 N_{\text {corr }}$ values for various water-soluble polymers ${ }^{\mathrm{a}}$

\begin{tabular}{lrlr}
\hline Polymer & $N_{\text {corr }}$ & Polymer & $N_{\text {corr }}$ \\
\hline Poly(acrylic acid sodium salt) & 5.9 & Polyacrylamide & 2.2 \\
Poly(L-lysine hydrobromide) & 5.5 & Poly(N-isopropylacrylamide) & 1.6 \\
Poly(allylamine hydrochloride) & 5.1 & Poly(ethylene glycol) & 0.7 \\
Poly(L-glutamic acid sodium & 4.6 & Poly(1-vinyl-2-pyrrolidone) & 0.7 \\
salt) & & & \\
Poly(acrylic acid) & 2.3 & Poly(phosphobetaine) (PMPC) & -0.7 \\
Poly(carboxymethyl betaine) & -0.3 & Poly(sulfopropyl betaine) (PSPB) & 1.2 \\
(PCMB) & & &
\end{tabular}

accumulated from refs $7-11$

a<smiles></smiles>

b<smiles>CC(C)(C)CC(C)(C)C(=O)NCCC[N+](C)(C)CCCS(=O)(=O)[O-]</smiles>

C<smiles>CC(C)(C)CC(C)(C(=O)OCC[N+](C)(C)CC(=O)[O-])C(C)(C)C</smiles>

Scheme 1 Chemical structures of zwitterionic polymers. Poly (phosphobetaine) (PMPC), poly(sulfopropyl betaine) (PSPB, $m=3$ ) and poly (carboxymethyl betaine) (PCMB). system, the $N_{\text {corr }}$ value was the smallest when the content of positively charged and negatively charged monomer residues in the copolymer are comparable, which indicates the importance of charge neutralization on the structure of vicinal water. These results are most likely due to the counteraction of the electrostatic hydration effect by the proximity between the cationic and anionic groups.

The partially positively charged hydrogen atoms of water are attracted to the negatively charged ions in solution, and the positively charged ions attract the partially negative oxygen atom of water to create hydration shells. ${ }^{17}$ When the cation and anion are in close proximity, the orientation of the hydrating water molecules may be greatly disturbed, resulting in a collapse of electrostriction.

The inertness of the zwitterionic polymers to the water structure appears to be unaffected by the positions of the cationic and anionic groups in the side chain (i.e., whether the positively charged group was closer to the main chain compared with the negatively charged group (PCMB and PSPB) or not (PMPC)). The charge neutralization is a decisive factor in the influence of these polymers on the water structure.

(3) In addition, we investigated the structure and H-bonding of water in aqueous solutions of various $\alpha$-amino acids using the same technique and found that the $\mathrm{H}$-bonded network structure of water in an aqueous solution of amino acids possessing a zwitterionic structure was not substantially affected by the hydrophilicity and hydrophobicity of the side groups. ${ }^{18}$ Moreover, the $N_{\text {corr }}$ values for $\alpha, \omega$-amino acids indicated that the $N_{\text {corr }}$ value for glycine was slightly positive. As the number of methylene groups between the carboxylate and ammonium groups increased, the $N_{\text {corr }}$ value gradually increased (Figure 3). ${ }^{19}$ These results suggest that the species with proximal anionic and cationic groups do not significantly disturb the H-bonded network structure of water as discussed above.

Because hydrophobic hydration around nonpolar methylene groups induces the formation of a local cage-like structure (clathrate), the results shown in Figure 3 appear to be intuitively strange. Notably, the solubility of $\alpha, \omega$-amino acid increased as the number of methylene groups $(n)$ increased (solubility in $100 \mathrm{ml}$ of water: glycine $(n=1)$, $25 \mathrm{~g}$ : $\beta$-alanine $(n=2), 55.5 \mathrm{~g}: \gamma$-butyric acid $(n=3)$ and $\varepsilon$-aminocaproic acid $(n=5)$, freely soluble), ${ }^{20}$ suggesting that the ionic properties of carboxylate and ammonium groups (i.e., their affinity for water molecules) increased with an increase in the distance between the oppositely charged ionic groups, which may partially decrease the formation of a cage-like structure of water molecules around the methylene groups. The trend in the $N_{\text {corr }}$ values obtained for

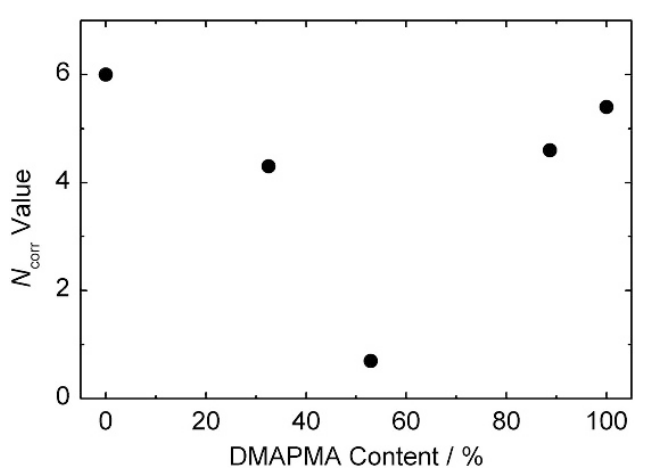

Figure $2 N_{\text {corr }}$ values for amphoteric polymers with various contents of negatively and positively charged monomers (methacrylic acid and 3-(dimethylamino)propyl methacrylamide), respectively. Poly(MA-r-DMAPMA). ${ }^{16}$ 


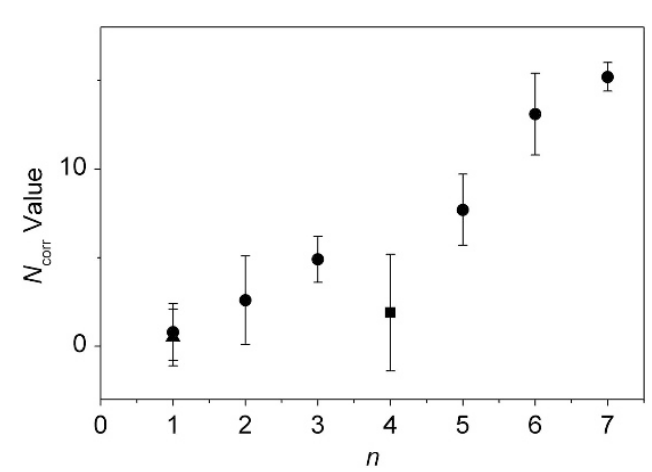

Figure $3 \mathrm{~N}_{\text {corr }}$ values for various $\alpha, \omega$-amino acids at $25^{\circ} \mathrm{C}$. Molar fraction $\left(p_{x}\right)=0.01 . \mathrm{H}_{3} \mathrm{~N}^{+}-\left(\mathrm{CH}_{2}\right)_{\mathrm{n}}-\mathrm{COO}^{-}$. ( $\left.\mathbf{\Delta}\right)$ betaine $\left(\mathrm{CH}_{3}\right)_{3}-\mathrm{N}^{+}-\mathrm{CH}_{2}-\mathrm{COO}^{-}$and $(\boldsymbol{\square}$ GlyGly. 19

the amino acid systems was consistent with the results obtained for zwitterionic polymers and amphoteric polymers described above in section 'Structure analyses of water in aqueous solutions containing water-soluble polymers' point (2).

\section{STRUCTURE ANALYSES OF WATER SORBED INTO POLYMER THIN FILMS}

Many types of polymer materials are used in the solid state. Therefore, structure analyses of water sorbed (adsorbed and absorbed) into water-insoluble polymers are needed. These analyses have been carried out by transmission and attenuated total reflection infrared (ATR-IR) spectroscopies. $^{21-23}$ At the early stage of water sorption, water is preferentially bound to polymer materials via hydrogen bonds between water molecules and polar groups in the polymer materials. Using the transmission method, it was found that the $\mathrm{O}-\mathrm{H}$ stretching spectra of adsorbed water depends on the H-bonding site of the polymers, and the absorption peak for water strongly $\mathrm{H}$-bonded with the polymer shifted to a lower frequency (Figure 4). ${ }^{22}$ This result is consistent with the trend previously reported by Kusanagi. ${ }^{24}$

The ATR-IR method is advantageous for the investigation of the diffusion process of water molecules in a polymer solid and the swelling process of polymer. ${ }^{25}$ In addition, from the difference ATRIR spectra of (1) water within a polymer film saturated with water vapor and (2) water sorbed into a polymer film onto which a water droplet was placed (i.e., (2) - (1)), the $\mathrm{O}-\mathrm{H}$ stretching band of water within the polymer matrix (secondary hydrating water) can be extracted.

We found that the water that secondarily hydrates films consisting of zwitterionic copolymers (poly(CMB-r-BMA), poly(MPC- $r$-BMA) and poly(SPB- $r$-BMA) (BMA, $n$-butyl methacrylate)), amphoteric copolymers (poly(MA-r-DMAPMA- $r$-BMA)) and nonionic poly(2-methoxyethyl acrylate) (PMEA), which exhibit excellent anticoagulant properties, was similar to the structure of bulk water (Figure 5). ${ }^{13,23,26,27}$ The results obtained by infrared spectroscopy in polymer thin film systems were consistent with the trend obtained using Raman spectroscopy to study aqueous polymer solutions (section 'Structure analyses of water in aqueous solutions containing water-soluble polymers' point (2)).

The structure of water sorbed into copolymer films consisting of various MEA and HEMA (2-hydroxyethyl methacrylate) amounts was also investigated by ATR-IR spectroscopy (Scheme 2). ${ }^{28}$ The apparent extinction coefficient of the $\mathrm{O}-\mathrm{H}$ stretching band of sorbed water $\left(\varepsilon_{\mathrm{OH}}\right)$ was calculated from the amount of sorbed water obtained by

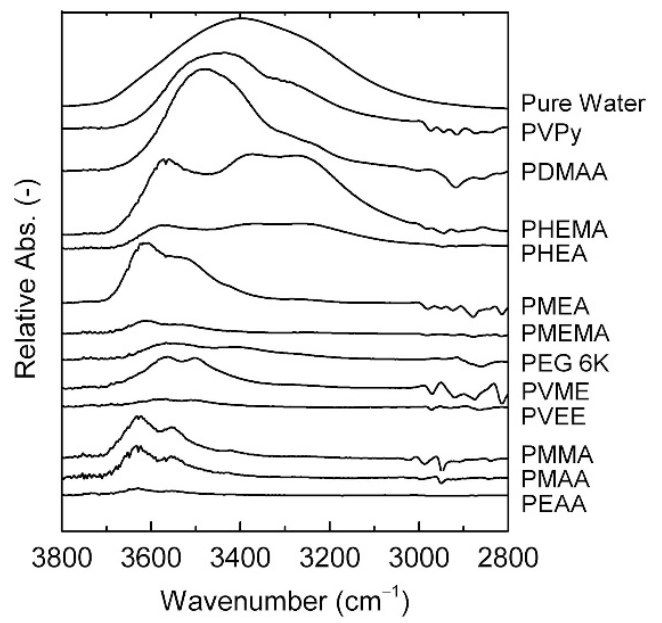

Figure $4 \mathrm{O}-\mathrm{H}$ stretching band for water sorbed into various polymer thin films $830 \mathrm{~s}$ after onset of contact with humid air (RH 55\%) (transmission mode). PDMAA, poly( $N, N$-dimethyl acrylamide); PEAA, poly(ethyl acrylate); PEG $6 \mathrm{~K}$, poly(ethylene glycol) ( $M_{\mathrm{w}}$ 6000); PHEA, poly(2-hydroxyethyl acrylate); PHEMA, poly(2-hydroxyethyl methacrylate); PMAA, poly(methyl acrylate); PMEA, poly(2-methoxyethyl acrylate); PMEMA, poly(2-methoxyethyl methacrylate); PMMA, poly(methyl methacrylate); PVEE, poly(vinyl ethyl ether); PVME, poly(vinyl methyl ether); and PVPy, poly(1-vinyl-2pyrrolidone). 22

thermogravimetric analysis and the band area obtained by ATR-IR measurement (equation (5); Figure 6).

$$
\varepsilon_{\mathrm{OH}}=\left(\int \mathrm{abs}_{v} \mathrm{~d} v\right) /\left(c d_{\mathrm{e}}\right)
$$

where $v$ is the wavenumber, $\operatorname{abs}_{v}$ is the absorbance at $v, c$ is the concentration of water in the polymer matrix, and $d_{\mathrm{e}}$ is the effective path length.

When polymers initially come into contact with liquid water, the $\varepsilon_{\mathrm{OH}}$ values drastically changed based on the MEA content. When the content of MEA was high (70-100 mol\%), the $\varepsilon_{\mathrm{OH}}$ values for water sorbed into the polymers in contact with liquid water were five to eight times larger than those for water sorbed into polymers in contact with water vapor. However, at a lower MEA content ( $<60 \mathrm{~mol} \%)$, the $\varepsilon_{\mathrm{OH}}$ value of water sorbed into polymers was equal to that in contact with water vapor. The number of platelets adhering to these copolymer films was strongly suppressed as the MEA content increased. ${ }^{29}$

In general, hydrogen-bonding environments substantially affect the $\varepsilon_{\mathrm{OH}}$ value of water molecules as follows: (1) the $\varepsilon_{\mathrm{OH}}$ value decreased due to the disruption of water clusters and hydration but increased by their formation, and (2) the $\varepsilon_{\mathrm{OH}}$ value of a water cluster is five times and 10 times or more larger than those of ordinary hydrating water and monomeric water (gaseous state), respectively. 3,4

These results suggest that the structure of water sorbed into the matrix of copolymers containing MEA residues is similar to that of bulk water, which is consistent with the results for zwitterionic copolymer films. The results also suggest that there is a strong correlation between the anticoagulant activity of the copolymer film and $\varepsilon_{\mathrm{OH}}$ of sorbed water. 
a

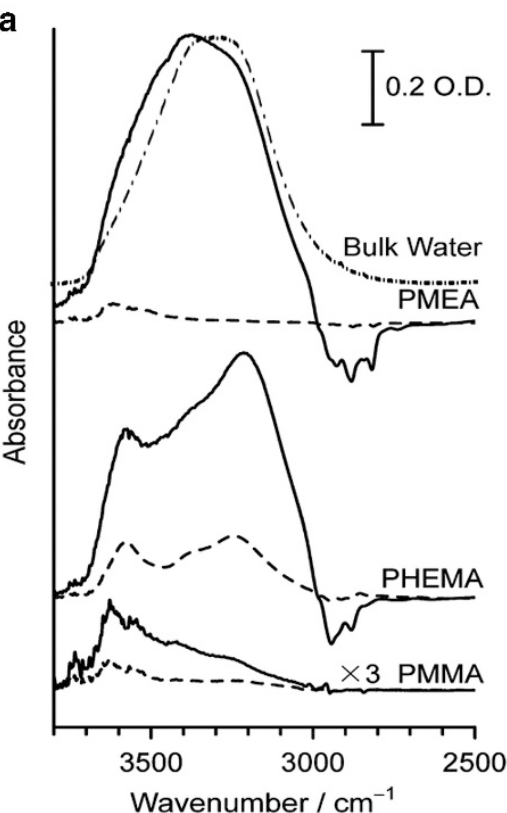

b

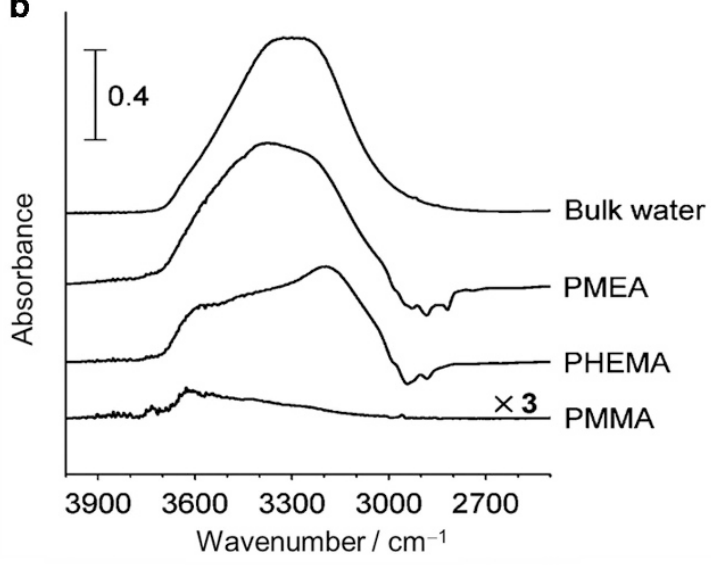

Figure 5 (a) Infrared spectra of water sorbed into various polymer films and (b) their difference spectra for sorbed water. PMMA, poly(methyl methacrylate); PHEMA, poly(2-hydroxyethyl methacrylate) and PMEA, poly(2-methoxyethyl acrylate). The solid line in a corresponds to polymer films in contact with liquid water, and the broken line corresponds to contact with water vapor. The spectra for bulk water were newly obtained using the ATR-IR method. ATR-IR, attenuated total internal reflectance-infrared. ${ }^{23}$

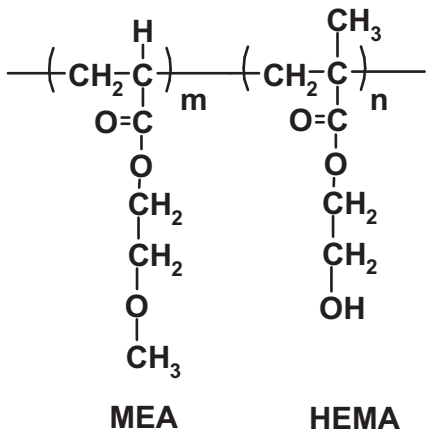

Scheme 2 Chemical structure of a copolymer of MEA and HEMA.

\section{STRUCTURE ANALYSES OF WATER AT POLYMER-LIQUID WATER INTERFACES}

The surface of many polymer materials is the functional area, except for structural polymer materials where a high degree of mechanical strength is required. Therefore, surface properties dominate the usability of polymer materials. Raman spectroscopy, which is described in section 'Structure analyses of water in aqueous solutions containing water-soluble polymers', has been used to determine the effect of water-soluble polymers on the hydrogen-bonded network structure of water in aqueous solutions. Infrared spectroscopy, which is described in section 'Structure analyses of water sorbed into polymer thin films', is used to determine the structure of water within solid polymer materials. Therefore, the use of these methods cannot provide 'direct' information regarding the water at 'material-water interfaces', which is strongly influenced by interactions with the polymer materials or other co-existing compounds. We have only been speculating about the structure of interfacial water using information from the water structure within solutions and films.

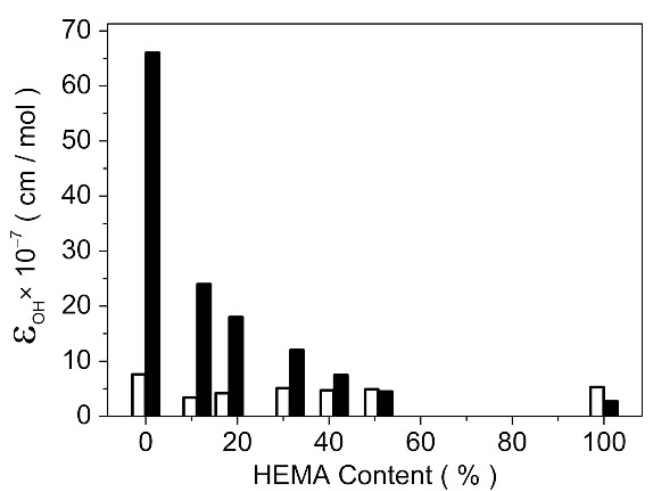

Figure 6 Apparent molecular extinction coefficient for water sorbed into polymer films with various contents of HEMA (2-hydroxyethyl methacrylate) and MEA (2-methoxyethyl acrylate) obtained using equation (5). The white bar represents the polymer film in contact with water vapor, and the black bar represents the difference spectra obtained by subtraction of the spectra of water sorbed into polymer film contacting water vapor from the spectra contacting liquid water. ${ }^{28}$

Therefore, we used the SFG method, which has a high interfacial sensitivity, to analyze interfacial water. The SFG spectra are expected to provide insight into the degree of orientation of the water molecules at the interfaces of solid materials and liquid water. Observations have been carried out using a homodyne system but not a phase-sensitive heterodyne system because the latter cannot be technically applied at present to liquid water-solid polymer interfaces that have a much larger degree of roughness than the size of the studied polymer molecules.

All of the studied systems were constructed on a flat surface consisting of a semi-cylindrical fused quartz prism. The systems include (i) various polymer brushes prepared using surface-initiated 


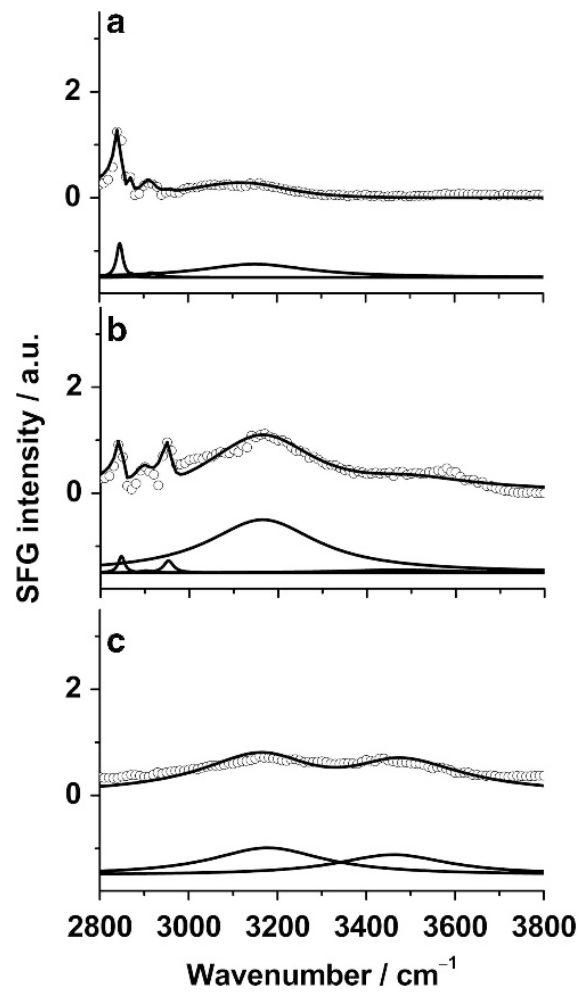

Figure 7 SFG spectra of the $\mathrm{O}-\mathrm{H}$ stretching region for various polymer films $48 \mathrm{~h}$ after onset of immersion in pure water. (a) Poly(CMB- $r$-BMA), CMB 29 mol\%; (b) Poly(MA-r-BMA), MA $29 \mathrm{~mol} \%$ and (c) Poly(DMAEMA-r-BMA), DMAEMA $28 \mathrm{~mol} \%$. Polarization combinations of SFG, visible and IR were $s, s$ and $p$, respectively. BMA, $n$-butyl methacrylate; CMB, carboxymethyl betaine; DMAEMA, 2-(dimethylamino)ethyl methacrylate; IR, infrared; MA, methacrylic acid; SFG, sum frequency generation. ${ }^{30}$

atom transfer radical polymerization or surface-initiated reversible addition-fragmentation chain transfer polymerization, (ii) copolymer thin films prepared by the dipping method and (iii) a self-assembled monolayer (SAM) of terminally functionalized silane coupling agents.

The SFG spectra in the $3000-3800 \mathrm{~cm}^{-1}$ range, which corresponds to $\mathrm{O}-\mathrm{H}$ stretching of interfacial water, contained two broad peaks at $\sim 3200 \mathrm{~cm}^{-1}$ and $\sim 3400 \mathrm{~cm}^{-1}$, as shown in Figure $7 .{ }^{30}$ The former peak is assigned to the symmetric $\mathrm{O}-\mathrm{H}$ stretching mode of tetrahedrally coordinated water molecules (strongly $\mathrm{H}$-bonded water molecules), and the latter peak is assigned to the symmetric $\mathrm{O}-\mathrm{H}$ stretching mode of asymmetrically $\mathrm{H}$-bonded water molecules (i.e., molecules with one $\mathrm{H}$ atom strongly bonded and the other $\mathrm{H}$ atom weakly bonded to neighboring molecules (weakly $\mathrm{H}$-bonded water molecules)). ${ }^{31,32}$ These peaks were very broad due to the strong coupling of neighboring water molecules and Fermi resonance with the $\mathrm{HOH}$ mode. ${ }^{4}$ The peaks were separated into two bands using Microcal Origin in our group.

The results obtained for systems (i)-(iii) described above can be summarized as follows:

(1) At the interfaces between liquid water and polymer brushes composed of comparative contents of positively and negatively charged monomers, the intensities of the SFG signal of the $\mathrm{O}-\mathrm{H}$ stretching region were smaller than those corresponding to the interfaces between liquid water and the corresponding lopsidedly charged polymer brushes. ${ }^{33}$ In addition, the SFG intensity of the $\mathrm{O}-\mathrm{H}$ stretching region at the interface of liquid water and the

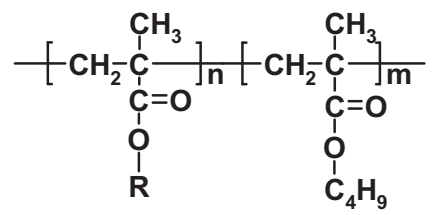

R:
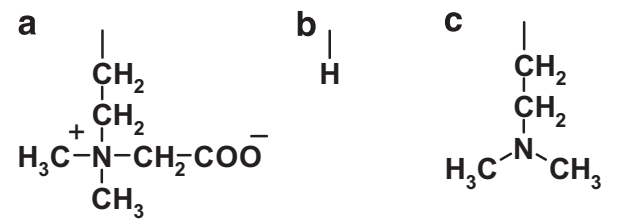

Scheme 3 Chemical structures of various types of copolymers. (a) Poly(CMB$r$-BMA), (b) Poly(MA-r-BMA) and (c) Poly(DMAEMA-r-BMA).

SAM of an ATRP initiator was larger than that of a zwitterionic polymer brush surface, indicating that the strong orientation of the interfacial water may be due to electrostatic or hydrophobic hydration. ${ }^{34}$

(2) Similarly, at the interfaces between liquid water and thin films composed of an apolar monomer ( $n$-butyl methacrylate, BMA) and zwitterionic monomer $(\mathrm{CMB})$ residues $(\sim 7: 3)$, the intensities of the SFG signal in the $\mathrm{O}-\mathrm{H}$ stretching region were smaller than those at the interfaces between liquid water and the corresponding lopsidedly charged copolymer thin films and PBMA thin film (Scheme 3, Figure 7 and Table 2). ${ }^{30}$ Contact angle measurements of an air-in-water system indicated that the surface of a zwitterionic poly(CMB- $r$-BMA) film was quickly equilibrated upon immersion in liquid water. However, the surfaces of positively charged poly(DMAEMA- $r$-BMA) and negatively charged poly(MA-r-BMA) films equilibrated more slowly, and even $48 \mathrm{~h}$ after the onset of immersion in liquid water, the lopsidedly charged copolymer films were not completely equilibrated. Therefore, the results indicate that the zwitterionic copolymer film did not induce a significant orientation in the interfacial water compared with the lopsidedly charged copolymer film.

(3) The same trend was observed for the SAM of a zwitterionic head group-carrying silane coupling agent or mixed SAM composed of comparative contents of positively and negatively charged silane coupling agents. ${ }^{35,36}$

Recently, phase-sensitive SFG spectroscopic analysis has revealed that water molecules at water/phospholipid interfaces are preferentially oriented on the basis of the electrostatic potential imposed by the phospholipids. ${ }^{37}$ Zwitterionic phosphatidylethanolamine and phosphatidylcholine exhibit weaker water-orientating capability relative to negative phospholipids, which is consistent with trend observed for charge-neutralized polymer systems in our group.

Using the same technique, the orientation of water molecules at phosphatidylcholine-water interfaces has been investigated in detail. ${ }^{38}$ The water surrounding the positively charged choline group forms weaker $\mathrm{H}$-bonds with a net $\mathrm{H}$-down orientation, and the water associated with the negatively charged phosphate group is strongly $\mathrm{H}$-bonded with net $\mathrm{H}$-up orientation.

The water molecules at the shortest distance from the phosphoryl choline groups might be affected by ionic groups. A huge number of water molecules from the secondary hydration layer may be affected by both negatively and positively charged groups, resulting in weaker 
Table 2 Intensity of SFG signal for the $\mathbf{O}-\mathrm{H}$ stretching region at the interfaces of liquid water and copolymer films ${ }^{\mathrm{a}}$

\begin{tabular}{|c|c|c|}
\hline \multirow[b]{2}{*}{ Sample } & \multicolumn{2}{|c|}{ Total } \\
\hline & $O h^{\mathrm{b}}$ & $4 h^{\mathrm{b}}$ \\
\hline PolyBMA & $130.2(0.58)$ & $166.0(0.74)$ \\
\hline Poly(CMB-r-BMA) & $108.4(0.48)$ & $50.4(0.22)$ \\
\hline Poly(MA-r-BMA) & $151.4(0.67)$ & $225.1(1.00)$ \\
\hline Poly(DMAEMA-r-BMA) & $72.6(0.32)$ & $158.4(0.71)$ \\
\hline
\end{tabular}

Abbreviations: BMA, $n$-butyl methacrylate; CMB, carboxymethyl betaine; DMAEMA,

2-(dimethylamino)ethyl methacrylate; MA, methacrylic acid; SFG, sum frequency generation.

aThe values in parentheses are peak areas normalized by the peak area for a bare fused

quartz prism.

'Time period after onset of immersion in liquid water. Data were taken from ref. 30.

orientation than that of primary hydration water. Phase-sensitive (heterodyne-detected) SFG can provide valuable insight into the 'direction' of orientation (hydration), and conventional (homodynedetected) SFG can provide information regarding the 'degree' of orientation.

It is important to note that both (i) charge-neutralized polymer brushes and (ii) zwitterionic copolymer thin films discussed in this section suppressed the nonspecific adsorption and adhesion of proteins and cells, respectively. For charge-neutralized SAMs (zwitterionic and amphoteric ones) (iii), the adsorption of proteins was also suppressed but the adhesion of cells was not effectively suppressed, which was most likely due to the rigidity of the solid materials that induced nonspecific adhesion of cells. These results are owing to the stronger contractile forces via the actin-myosin interaction and cytoskeleton organization being generated by the attachment of cells to stiffer matrices, which leads to the formation of larger and more stable focal adhesion complexes. ${ }^{39}$ Therefore, the reason for the extensive use of polymer materials in biomedical fields has been demonstrated.

The results described in sections 'Structure analyses of water in aqueous solutions containing water-soluble polymers', 'Structure analyses of water sorbed into polymer thin films' and 'Structure analyses of water at polymer-liquid water interfaces' strongly suggest that the driving force for the biocompatibility of charge-neutralized polymers (including brushes and thin films), SAMs and nonionic PMEA may be due to the small influence on interfacial water. Before the adhesion of cells to a polymer material, proteins adsorb to the surface of the material. At the interfaces between liquid water and polymers that determine the orientation of water, proteins collide and oriented (strongly hydrated) water molecules are released, resulting in an increase in entropy. Therefore, adsorption of proteins and subsequent adhesion of cells occur. The increase in entropy will be smaller at the interfaces between liquid water and polymers that do not induce strong orientation of vicinal water molecules. This behavior indicates an important guideline for the development of polymer materials: Materials that suppress the orientation of interfacial water will be biocompatible.

\section{ANALYSES OF FREEZING AND MELTING BEHAVIORS OF WATER SORBED INTO POLYMER MATERIALS}

We also analyzed the freezing and melting behavior of water sorbed into polymer materials between room temperature and $140 \mathrm{~K}$ using temperature-variable infrared spectroscopy. ${ }^{40-44}$ Few studies of this nature have been reported owing to the complicated procedures. PMEA, poly(tetrahydrofurfuryl acrylate) (PTHFA), poly(methyl a Cooling rate $=0.25 \mathrm{~K} \cdot \mathrm{min}^{-1}$ 265230210
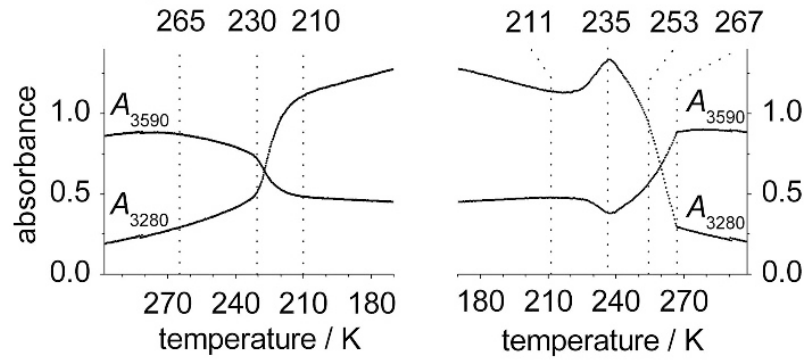

b Cooling rate $=5.0 \mathrm{~K} \cdot \mathrm{min}^{-1}$ $260 \quad 211$

211235253267
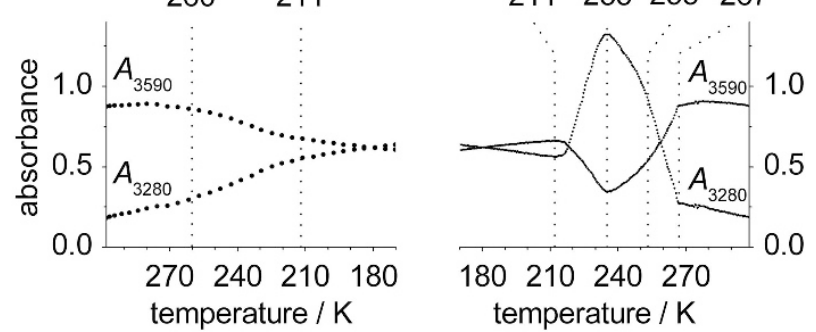

Figure 8 Effect of cooling rate on the freezing/melting behavior of water sorbed into PMEA thin films (water content, $3.2 \mathrm{wt} \%$ ). IR spectroscopic data. Cooling rate: (a) $0.25 \mathrm{~K} \mathrm{~min}^{-1}$ and (b) $5.0 \mathrm{~K} \mathrm{~min}^{-1}$. IR, infrared; PMEA, poly(2-methoxyethyl acrylate). ${ }^{41}$

acrylate) (PMAA), PBMA, poly(2-ethoxyethyl acrylate) (PEEA), poly (2-ethoxyethyl methacrylate) (PEEMA) and polystyrene (PS) were investigated. ${ }^{43}$

A previous study using differential scanning calorimetry (DSC) indicated that there were no state changes (freezing and melting) of water incorporated in these polymer materials at a low water content. In addition, the presence of water that exhibited a so-called 'cold crystallization' (recrystallization) phenomenon (upon rapid cooling and subsequent gradual heating, a portion of the water freezes at a temperature of approximately $-45^{\circ} \mathrm{C}$.) inside the PMEA hydrogel has been suggested on the basis of DSC measurements. ${ }^{29}$

Temperature-dependent infrared spectroscopic measurements of PMEA films have been performed (Figure 8). ${ }^{41}$ Absorbances at 3590 $\mathrm{cm}^{-1}\left(A_{3590}\right)$ and $3280 \mathrm{~cm}^{-1}\left(A_{3280}\right)$ are indicative of monomolecular water hydrating the polymer chain and condensed water (ice $I_{h}$ ) in the polymer matrix, respectively. With rapid cooling (panel (b)), a steep increase in the value of $A_{3280}$ during cooling was not observed (left of panel (b)), indicating no crystallization of the sorbed water. During rewarming after rapid cooling (right of panel (b)), a substantial increase in $A_{3280}$ in the temperature range of $211-235 \mathrm{~K}$ indicated a large degree of recrystallization of the sorbed water. A synchronous decrease in $A_{3590}$ with an increase in $A_{3280}$ suggests that the glassy water did not recrystallize but the monomolecular water did. However, with slow cooling (panel (a)), a steep and large increase in $A_{3280}$ at $230 \mathrm{~K}$ (left of panel (a)) was observed during cooling. During rewarming after the slow cooling, a smaller increase in $A_{3280}$ compared with that after rapid cooling was observed. These results indicate that the amount of recrystallized water (and also crystallized water) strongly depends on the cooling rate of the sample. ${ }^{41}$ Therefore, the amount of so-called 'intermediate water', which is often used to indicate the cold crystallization of water, cannot be unequivocally determined. 
In addition to PMEA, the recrystallization of water in various polymer matrices, such as PTHFA, PEEA, PEEMA, PMAA, PBMA and PS, has also been observed (Figure 9). However, no recrystallization was observed in the poly(n-butyl acrylate) (PBA) and PMMA matrices. ${ }^{43,44}$ The recrystallization temperature $\left(T_{\mathrm{rc}}\right)$ of water in the polymers that possess a relatively high $\mathrm{O}$ atom density and low glass transition temperature $\left(T_{\mathrm{g}}\right)$ (i.e., PMAA, PEEA, PEEMA, PTHFA and PMEA) increased as the $T_{\mathrm{g}}$ increased. However, the $T_{\mathrm{rc}}$ value of water in polymers, which possess a relatively high $T_{\mathrm{g}}$ and low $\mathrm{O}$ atom density (PBMA) or without $\mathrm{O}$ atoms (PS), decreased as the $T_{\mathrm{g}}$ increased. The former (PMAA, PEEA, PEEMA, PTHFA and PMEA) and the latter (PBMA and PS) cases strongly suggested that recrystallization depends on the strength of the electrostatic interactions

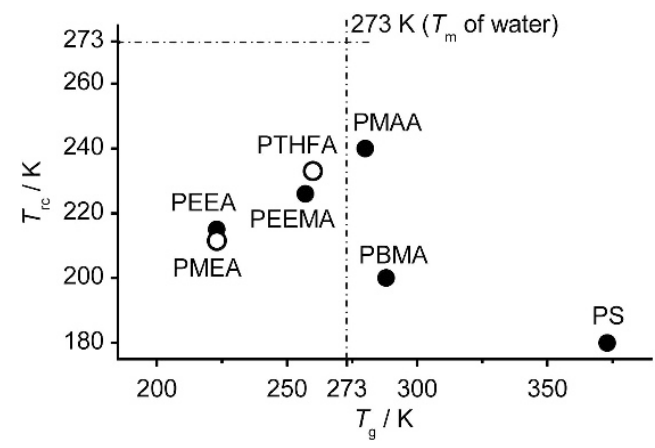

Figure 9 Relationship between temperature of recrystallization $\left(T_{\mathrm{rc}}\right)$ and glass transition temperature $\left(T_{\mathrm{g}}\right)$ of polymer materials. PBMA, poly $(n$-butyl methacrylate); PEEA, poly(2-ethoxyethyl acrylate); PEEMA, poly(2-ethoxyethyl methacrylate); PMAA, poly(methyl acrylate); PMEA, poly(2-methoxyethyl acrylate); PTHFA, poly(tetrahydrofurfuryl acrylate); PS, polystyrene. PMEA and PTHFA are anti-coagulants. ${ }^{43}$ between water molecules and the polymer (i.e., the affinity of water for the polymer chains) as well as the mobility of the polymer chains (i.e., $T_{\mathrm{g}}$ differences).

In general, the phenomenon has been ascribed to the crystallization of amorphous ice, which is produced in the cooling step, and the presence of recrystallization has been noted as a driving force for biocompatibility. ${ }^{29}$ Although ice growth in the heating process was observed by temperature-variable IR spectroscopy, the existence of amorphous ice could not be confirmed in the obtained IR spectra. The absorption band for amorphous ice is located at $\sim 3200 \mathrm{~cm}^{-1} .45$ However, we could not detect the presence of this absorption band during the cooling/heating processes. In addition, the cold crystallization phenomenon (increase in absorbance at $3280 \mathrm{~cm}^{-1}$ ) was associated with a decrease in primary hydration water (decrease in absorbance at $3590 \mathrm{~cm}^{-1}$ ), as discussed above (Figure 8). The recrystallization process does not involve devitrification of amorphous ice but does involve direct state changes of primary hydration water to ice without passing through a condensed phase, such as liquid water or glass state water.

In addition, because recrystallization phenomena were observed for PS, PBMA and PEEA, which are not biocompatible (Figure 9), the correlation between the presence of recrystallized water and the anticoagulant ability of PMEA and PTHFA should be carefully reconsidered.

The state changes of water in polymer-water systems are assumed to include only freezing/melting or nothing, without any relationship to whether the water content of the polymer was high or low or the polymer was soluble in water. This general understanding of the water structure is based on results from calorimetric analyses, such as DSC. Because the obtained data are relatively easy to interpret, DSC is one of the most frequently used methods to analyze the water structure in polymer-water systems, as described in the second paragraph of this a

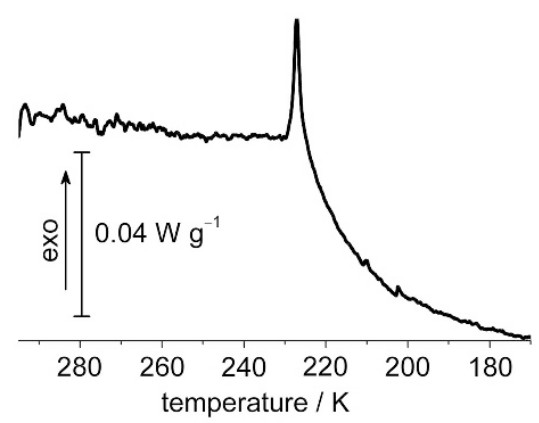

C

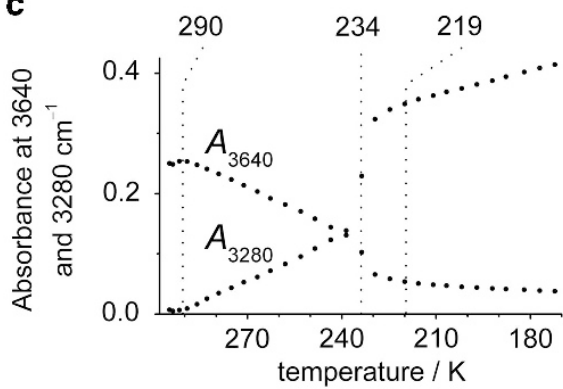

b

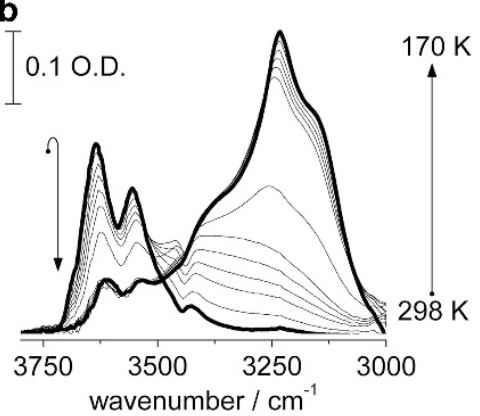

Figure 10 DSC and infrared spectroscopic data for water sorbed into a thin film of poly( $n$-butyl acrylate) (water content, $0.44 \%$ ) in the cooling step. (a) DSC data; (b, c) infrared data. DSC, differential scanning calorimetry. ${ }^{44}$ 
section. However, this ease of interpretation can be accepted only when the understanding stated above is applicable.

To confirm this point, the state changes of water sorbed into poly ( $n$-butyl acrylate) (PBA) were analyzed in detail using temperaturevariable IR spectroscopy and DSC. ${ }^{44}$ Unexpectedly, the experimental results indicated that the general understanding of water sorbed into a water-insoluble polymer, such as PBA, by vapor sorption is not always acceptable. The sorbed water can be condensed, crystallized and deposited during cooling (panels (b) and (c) in Figure 10) as well as sublimated, become molten and vaporized during heating (data not shown).

In addition, the condensation, deposition, sublimation and desorption of sorbed water in PBA, which was observed by IR spectroscopy, could not be detected by calorimetric analysis. The condensation processes (decrease in absorbance at $3640 \mathrm{~cm}^{-1}$ in the temperature range of $298 \sim 235 \mathrm{~K}$ ) indicated in panels (b) and (c) in Figure 10 were not detected by DSC (panel (a)) because the enthalpy of desorption is similar to the enthalpy of condensation, which occurs simultaneously. ${ }^{44}$ These results provide new insight into the water in non-water-soluble polymers and indicate the need for reconsideration of the general understanding of water in aqueous polymer solutions.

\section{CONCLUSION}

The series of studies described here can be linked to the molecular design of functional materials based on the structure analyses of vicinal water; therefore, the molecular design of biocompatible materials is included in this category. In addition, the sorption of water as well as the freezing and melting of sorbed water are important phenomena that can result in the degradation of polymer materials. Therefore, the in situ analytical procedure (i.e., temperature-variable IR spectroscopy) described in 'Analyses of freezing and melting behaviors of water sorbed into polymer materials' provides valuable insight for the improvement of durable polymer materials for use under normal and extreme conditions, such as in the polar region and in spacecraft. As a result, the analytical procedures introduced in this review are scientifically and industrially important for the development of polymer materials.

\section{CONFLICT OF INTEREST}

The author declares no conflict of interest.

\section{ACKNOWLEDGEMENTS}

The series of studies introduced in this review were pursued by collaboration with many researchers, who are listed below. I sincerely thank these researchers for their strong support. These researchers include Professor Yasushi Maeda (University of Fukui), Professor Makoto Gemmei-Ide (University of Toyama), Professor Kohji Ohno (Institute for Chemical Research, Kyoto University), Professor Tadashi Nakaji-Hirabayashi (University of Toyama), Professor Mitsuhiro Fukuda (Hyogo University of Education), Professor Makoto Kawagoe (Toyama Prefectural University), Professor Kohei Uosaki (NIMS), Dr Hidenori Noguchi (NIMS), Professor Masaru Tanaka (Kyushu University), Professor Akira Mochizuki (Tokai University) and Professor Kazuhiko Ishihara (University of Tokyo). In addition, I am grateful to all of the students previously and currently pursuing research in our laboratory for their continuous efforts to complete research studies.

1 Anfinsen, C. B. Principles that govern the folding of protein chains. Science 181, 223-230 (1973).

2 Terada, T., Inaba, T., Kitano, H., Maeda, Y. \& Tsukida, N. Raman spectroscopic study on water in aqueous solutions of temperature-responsive polymers: poly $(\mathrm{N}$ - isopropylacrylamide) and poly[N-(3-ethoxypropyl)-acrylamide]. Macromol. Chem. Phys. 195, 3261-3270 (1994).

3 Franks, F. in Water-A Comprehensive Treatise, Vol. 1 (ed. Franks, F.) (Plenum Press, New York, 1972)

4 Eisenberg, D. \& Kauzmann, W. The Structure and Properties of Water, (Clarendon Press, Oxford, 1969)

5 Hecht, D., Lee, L. \& Walters, L. Defining hydrophobicity: probing the structure of soluteinduced hydration shells by Fourier transform infrared spectroscopy. J. Am. Chem. Soc. 114, 4336-4339 (1992).

6 Walrafen, G. E. Raman spectral studies of the effects of temperature on water structure. J. Chem. Phys. 47, 114-126 (1967).

7 Georgiev, G. M., Kalkanjiev, T. K., Petrov, V. P., Nickolov, Z. \& Miteva, M. Concentration-dependence studies of Raman spectra of water by the method of selfdeconvolution. Chem. Phys. Lett. 103, 83-88 (1983).

8 Green, G. L., Lacey, A. R. \& Seats, M. G. Spectroscopic evidence for spatial correlations of hydrogen bonds in liquid water. J. Phys. Chem. 90, 3958-3964 (1986).

9 Terada, T., Maeda, Y. \& Kitano, H. Raman spectroscopic study on water in polymer gels. J. Phys. Chem. 97, 3619-3622 (1993).

10 Maeda, Y., Tsukida, N., Kitano, H., Terada, T. \& Yamanaka, J. Raman spectroscopic study of water in aqueous polymer solutions. J. Phys. Chem. 97, 13903-13906 (1993).

11 Kitano, H., Sudo, K., Ichikawa, K., Ide, M. \& Ishihara, H. Raman spectroscopic study on the structure of water in aqueous polyelectrolyte solutions. J. Phys. Chem. B 104, 11425-11429 (2000).

12 Kitano, H., Imai, M., Sudo, K. \& Ide, M. Hydrogen-bonded network structure of water in aqueous solution of sulfobetaine polymers. J. Phys. Chem. B 106, 11391-11396 (2002).

13 Kitano, H., Imai, M., Mori, T., Gemmei-Ide, M., Yokoyama, Y. \& Ishihara, K. Structure of water in the vicinity of phospholipid analogue copolymers as studied by vibrational spectroscopy. Langmuir 19, 10260-10266 (2003).

14 Kitano, H., Tada, S., Mori, T., Takaha, K., Gemmei-Ide, M., Tanaka, M., Fukuda, M. \& Yokoyama, Y. Correlation between the structure of water in the vicinity of carboxybetaine polymers and their blood-compatibility. Langmuir 21, 11932-11940 (2005).

15 Kitano, H., Takaha, K. \& Gemmei-Ide, M. Raman spectroscopic study on the structure of water in aqueous solution of amphoteric polymers. Phys. Chem. Chem. Phys. 8, 1178-1185 (2006).

16 Kitano, H., Nagaoka, K., Tada, S. \& Gemmei-Ide, M. Structure of water in the vicinity of amphoteric polymers as studied by Raman spectroscopy. J. Colloid Interface Sci. 313, 461-468 (2007).

17 Garrett, R. H. \& Grisham, C. M. in Biochemistry, (Saunders, Fort Worth, TX, 1995)

18 Ide, M., Maeda, Y. \& Kitano, H. Effect of hydrophobicity of amino acids on the structure of water. J. Phys. Chem. B 101, 7022-7026 (1997).

19 Kitano, H., Takaha, K. \& Gemmei-Ide, M. Raman spectroscopic study on the structure of water in aqueous solution of $\alpha, \omega$-amino acids. J. Colloid Interface Sci. 283, 452-458 (2005).

20 Merck Index, in An Encyclopedia of Chemicals, Drugs, and Biologicals. 13th edn Merck \& Co., Inc., (2001).

21 Ide, M., Yoshikawa, D., Maeda, Y. \& Kitano, H. The state of water at the surface of poly (ethyleneglycol) films examined by FT-IR. Langmuir 15, 926-929 (1999).

22 Ichikawa, K., Mori, T., Kitano, H., Fukuda, M., Mochizuki, A. \& Tanaka, M. Fourier transform infrared study on the sorption of water to various kinds of polymer thin films. J. Polym. Sci. Polym. Phys. 39, 2175-2182 (2001).

23 Kitano, H., Ichikawa, K., Fukuda, M., Mochizuki, A. \& Tanaka, M. The structure of water sorbed to polymethoxyethylacrylate film as examined by FT-IR spectroscopy. J. Colloid Interface Sci. 242, 133-140 (2001).

24 Kusanagi, H. \& Yukawa, S. Fourier transform infra-red spectroscopic studies of water molecules sorbed in solid polymers. Polymer 35, 5637-5640 (1994).

25 Sammon, C., Deng, C., Mura, C. \& Yarwood, J. Vibrational spectroscopic studies of the dynamics and perturbation of water in polymeric films. J. Mol. Lid. 101, 35-54 (2002).

26 Kitano, H., Tada, S., Mori, T., Takaha, K., Gemmei-Ide, M., Yokoyama, Y. \& Tanaka, M. Structure of water in sulfobetaine polymer films as revealed by ATR-IR. Macromol. Biosci. 5, 314-321 (2005)

27 Kitano, H., Nagaoka, K., Tada, S., Gemmei-Ide, M. \& Tanaka, M. Structure of water incorporated in amphoteric polymer thin films as revealed by FTIR spectroscopy. Macromol. Biosci. 8, 77-85 (2008).

28 Ide, M., Mori, T., Ichikawa, K., Kitano, H., Tanaka, M., Mochizuki, A., Oshiyama, H. \& Mizuno, W. Structure of water sorbed into poly(MEA-co-HEMA) films as examined by ATR - IR spectroscopy. Langmuir 19, 429-435 (2003).

29 Tanaka, M., Mochizuki, A., Ishii, N., Motomura, T. \& Hatakeyama, T. Study of blood compatibility with poly(2-methoxyethyl acrylate). Relationship between water structure and platelet compatibility in poly(2-methoxyethylacrylate-co-2-hydroxyethylmethacrylate). Biomacromolecules 3, 36-41 (2002).

30 Kondo, T., Nomura, K., Gemmei-lde, M., Kitano, H., Noguchi, H., Uosaki, K \& Saruwatari, Y. Structure of water at zwitterionic copolymer film-liquid water interfaces as examined by the sum frequency generation method. Colloids Surf. B Biointerfaces 113, 361-367 (2014).

31 Scherer, J. R. in Advances in Infrared and Raman Spectroscopy, Vol. 5 (eds Clark, R. J. H. \& Hester, R. E.) 149 (Heyden, Philadelphia, 1978)

$32 \mathrm{Du}, \mathrm{Q}$., Superfine, R., Freysz, E. \& Shen, Y. R. Vibrational spectroscopy of water at the vapor/water interface. Phys. Rev. Lett. 70, 2313-2316 (1993).

33 Kondo, T., Gemmei-Ide, M., Kitano, H., Ohno, K., Noguchi, H. \& Uosaki, K. Sum frequency generation study on the structure of water in the vicinity of an amphoteric polymer brush. Colloids Surf. B Biointerfaces 91, 215-218 (2012). 
34 Kondo, T., Nomura, K., Murou, M., Gemmei-Ide, M., Kitano, H., Ohno, K., Noguchi, H., Uosaki, K. \& Saruwatari, Y. Structure of water in the vicinity of a zwitterionic polymer brush as examined by sum frequency generation method. Colloids Surf. B Biointerfaces 100, 126-132 (2012).

35 Nomura, K., Nakaji-Hirabayashi, T., Gemmei-Ide, M., Kitano, H., Noguchi, H. \& Uosaki, K. Sum frequency generation analyses of the structure of water at amphoteric SAM-liquid water interfaces. Colloids Surf. B Biointerfaces 121, 264-269 (2014).

36 Nomura, K., Nakaji-Hirabayashi, T., Gemmei-Ide, M., Kitano, H., Noguchi, H. \& Uosaki, K. Sum frequency generation analyses of the structure of water at chargeneutralized SAM-liquid water interfaces. Polym. Prepr. Jpn 62, 984 (2013).

37 Chen, X., Hua, W., Huang, Z. \& Allen, H. C. Interfacial water structure associated with phospholipid membranes studied by phase-sensitive vibrational sum frequency generation spectroscopy. J. Am. Chem. Soc. 132, 11336-11342 (2010).

38 Mondal, J. A., Nihonyanagi, S., Yamaguchi, S. \& Tahara, T. Three distinct water structures at a zwitterionic lipid/water interface revealed by heterodyne-detected vibrational sum frequency generation. J. Am. Chem. Soc. 134, 7842-7850 (2012).

39 Ghibaudo, A. J., Saez, A., Trishet, L., Xayaphoummine, A., Browaeys, J., Silberzan, P., Buguin, A. \& Ladoux, B. Traction forces and rigidity sensing regulate cell functions. Soft Matter 4, 1836-1843 (2008).
40 Gemmei-Ide, M. \& Kitano, H. Spectroscopic evidence of phase transition of monomolecular water in solid polystyrene. J. Phys. Chem. B 112, 2764-2766 (2008).

41 Gemmei-Ide, M. \& Kitano, H. Recrystallization of water in a non-water-soluble polymer examined by Fourier transform infrared spectroscopy: poly(2-methoxyethylacrylate). J. Phys. Chem. B 112, 12863-12866 (2008).

42 Gemmei-Ide, M. \& Kitano, H. Crystal growth of ice $I_{h}$ by re-vapor-deposition and diffusion suppression of monomolecular water in a polymer solid: spectroscopic observation of phase transition of water sorbed into polystyrene. J. Phys. Chem. B 112, 13499-13502 (2008).

43 Gemmei-Ide, M., Ohya, A. \& Kitano, H. Recrystallization of water in non-water-soluble (meth)acrylate polymers is not rare and is not devitrification. J. Phys. Chem. B 116, 1850-1857 (2012).

44 Gemmei-Ide, M., Ohya, A. \& Kitano, H. Thermally latent water in a polymer matrix. J. Phys. Chem. B 114, 4310-4312 (2010).

45 Mallamace, F., Broccio, M., Corsaro, C., Faraone, A., Majolino, D., Venuti, V., Liu, L., Mou, C. Y. \& Chen, S. H. Evidence of the existence of the low-density liquid phase in supercooled, confined water. Proc. Natl Acad. Sci. USA 104, 424-428 (2007).

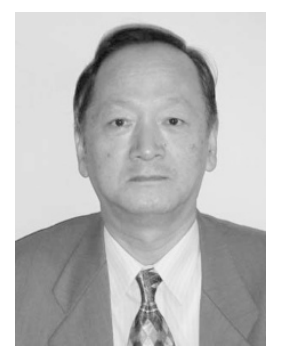

Professor Hiromi Kitano belongs to the Department of Applied Chemistry of the Graduate School of Science and Engineering at the University of Toyama. He received his PhD from Kyoto University in 1980 under the supervision of Professor Norio Ise of the Department of Polymer Chemistry. He joined the Kyoto University from 1978 to 1991 as an assistant professor and a lecturer. From April 1983 to March of 1984, he stayed at the University of Mainz, Germany. He joined the University of Toyama in 1991 as an associate professor and from 1992 as a professor. From January to March of 2004, he stayed at the University of Cambridge, United Kingdom. His studies include the structure analyses of water in the vicinity of polymer materials, development and application of biocompatible polymers, and analyses of molecular recognition processes at the interfaces of water-polymer materials. He was a recipient of The Award of the Society of Polymer Science, Japan (2013). 